\title{
Pressure gradient effect at distributed excitation of 3D TS waves by freestream and wall disturbances
}

\author{
Vladimir Borodulin, Andrey Ivanov, Yury Kachanov, Dmitry Mischenko*, and \\ Anna Fedenkova \\ Khristianovich Institute of Theoretical and Applied Mechanics SB RAS, 630090 Novosibirsk, Russia
}

\begin{abstract}
The present work is a continuation of previous experiments (carried out in the Blasius boundary layer) and devoted to quantitative investigation of influence of an adverse pressure gradient on two efficient mechanisms of excitation of 3D TS instability waves due to a distributed boundary layer receptivity to free-stream vortices. These mechanisms are associated with distributed scattering of 3D amplified free-stream vortices both on the natural boundary-layer nonuniformity (on smooth surface) and on 2D surface nonuniformities (waviness). The corresponding detailed hotwire measurements were carried out in a self-similar boundary layer with Hartree parameter $\beta_{\mathrm{H}}=-0.115$ in a wide range of the problem parameters. Complex values of quantitative characteristics of the physical phenomenon under study (the distributed receptivity coefficients) are evaluated by based on the obtained experimental data. It is found that the adverse pressure gradient leads to reduction of efficiency of the investigated vortexroughness receptivity mechanism.
\end{abstract}

\section{Introduction}

The present experiments are devoted to quantitative investigation of adverse-pressuregradient effects on the mechanisms of excitation of three-dimensional Tollmien-Schlichting (TS) waves due to distributed (in the streamwise direction) boundary layer receptivity to free-stream vortices. These mechanisms are associated with distributed scattering of threedimensional unsteady free-stream vortices both on the natural boundary layer nonuniformity (smooth surface) and on 2D surface nonuniformities (waviness). The receptivity and the boundary-layer instability mechanisms affect simultaneously on downstream evolution of TS-waves and provide the possibility of much faster growth of the latter compared to the growth rates associated with the linear instability mechanism.

The majority of previous studies were theoretical and they dealt with $2 \mathrm{D}$ problems. The first experimental estimations of the distributed vortex receptivity coefficients were carried out in [1] for a two-dimensional problem. Experiments [2] were devoted to investigation of $3 \mathrm{D}$ vortex receptivity problem in the Blasius boundary layer. These experiments gave for the first time systematic information on the distributed excitation of $3 \mathrm{D}$ TS-waves by

* Corresponding author: d.a.mischenko@itam.nsc.ru 
freestream vortices in presence of 2D surface roughness. The values of the distributed receptivity coefficients were obtained there in a broad range of the problem parameters. The present work is a continuation of experiments [2] and devoted to a systematic quantitative investigation of influence of an adverse pressure gradient on the distributed vortical receptivity mechanisms mentioned above. The present experiments were performed in a self-similar boundary layer with Hartree parameter $\beta_{\mathrm{H}}=-0.115$.

\section{Experiment setup and evolution of exited TS-modes}

The measurements were carried out in a low-turbulence wind tunnel T-324 of ITAM SB RAS in a boundary layer of a high-precision experimental model consisted of a flat plate $(1485 \times 1000 \times 10 \mathrm{~mm})$ and a wall bump of a special adjustable shape mounted on the windtunnel ceiling just above the plate. The shape of the wall bump provided formation on the flat plate in the region of main measurements of a self-similar flow with Hartree parameter $\beta_{\mathrm{H}}=-0.115$. The boundary-layer edge velocity $U_{\mathrm{e}}$ varied from 8.9 to $8.6 \mathrm{~m} / \mathrm{s}$ in the region of the streamwise coordinate $x=350$ to $620 \mathrm{~mm}$ having its origin at the plate leading edge. The corresponding Reynolds numbers $\operatorname{Re}^{*}=U_{e} \delta_{1} / v$ varied between 837 and 1174 (here $\delta_{1}$ is the boundary layer displacement thickness and $v$ is the air kinematic viscosity). The $3 \mathrm{D}$ freestream vortices were generated by a vibrating wire mounted normally to the flat-plate surface upstream of its leading edge. The 2D surface nonuniformities of sinusoidal shape were created by special thin-film patches applied onto the plate surface. These patches were manufactured by a special high-precision technology and had rated parameters: the streamwise wavelength $\lambda_{\mathrm{sx}}$ and the amplitude $h_{\mathrm{s}}$. Thorough measurements of the boundary layer and freestream disturbances were carried out by a single hot-wire probe in several regimes (see the Table 1). In some of these regimes, the problem parameters (such as the disturbance frequency $f$, the streamwise surface-waviness wavelength, and the propagation angle $\theta$ of the excited TS-waves) were chosen in a way to provide the satisfaction of conditions of the resonance of streamwise wavenumbers of the excited TS-modes, freestream vortices, and surface nonuniformities [2].

Table 1. Main regimes of measurements.

\begin{tabular}{ccccc}
\hline Regime name & Roughness \# & $f[\mathrm{~Hz}]$ & $\lambda_{\mathrm{sx}}[\mathrm{mm}]$ & $h_{\mathrm{s}}[\mu \mathrm{m}]$ \\
\hline$R 1 f 1$ & 1 & 53.7 & 85.7 & 177 \\
$R 3 f 2$ & 3 & 81.4 & 63.5 & 199 \\
$R 5 f 2$ & 5 & 81.4 & 69.8 & 215 \\
$R 6 f 3$ & 6 & 109.1 & 51.8 & 179 \\
\hline
\end{tabular}

The characteristics of the TS-modes' linear evolution on the wavy surfaces were measured in additional series of experiments in all main studied regimes in absence of freestream disturbances. These measurements were necessary for obtaining quantitative characteristics of the studied mechanism of excitation of the instability modes - the vortex/roughness distributed receptivity coefficients. In these additional experiments the wave-trains of controlled 3D TS-waves were excited by special point disturbance source (of blowing/suction type) mounted in the beginning of the experimental model.

The experiments have shown that a controlled antisymmetric vortex street is produced in the wake of the vibrating wire and leads to a rather efficient excitation of wave trains consisted of 3D TS-modes. The effective excitation of 3D TS-waves by the point disturbance source was also achieved in the additional (stability) experiments. In the two experiments, a number of spanwise profiles of amplitudes and phases of excited boundary layer disturbances were measured at various streamwise positions. Streamwise distributions 
of amplitudes and phases of the excited TS-modes were decomposed into the spanwisewavenumber spectra. It was found that due to the action of the distributed receptivity mechanisms, the amplitudes of the excited TS-waves are able to grow much faster then those generated in the case of action of the linear instability mechanism only. Fig. 1 illustrates a typical picture of comparison of streamwise evolutions of the boundary layer disturbances ( $a$ - amplitudes, $b$ - phases) in the main (receptivity) and in the additional (stability) experiments.

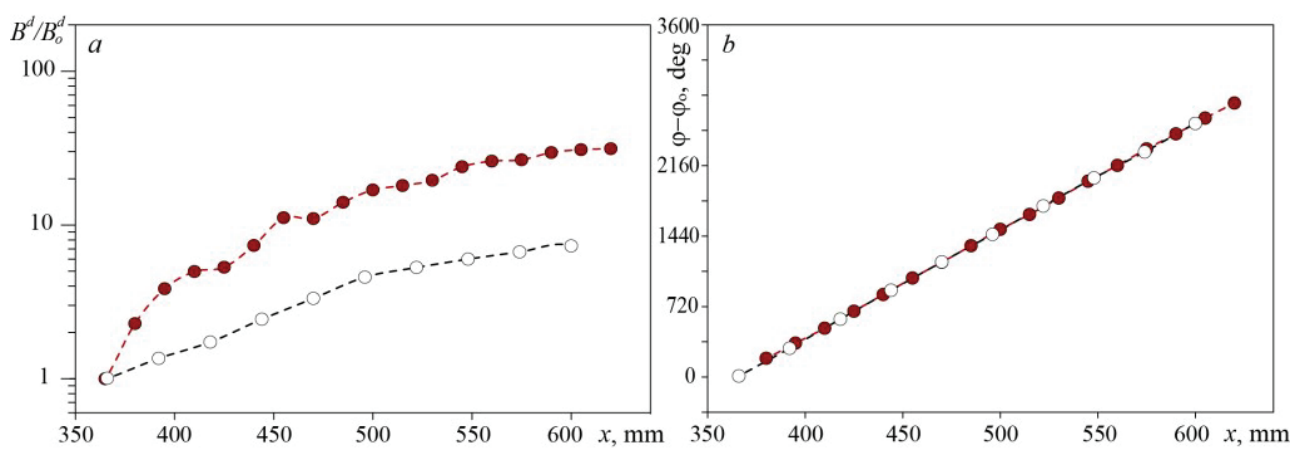

Fig. 1. Comparison of streamwise evolutions of amplitudes $(a)$ and phases $(b)$ of oblique TS-waves generated in receptivity experiment (dark points) and in additional stability experiment (open points). Regime $R 6 f 3, \theta=41 \mathrm{deg}$.

Qualitatively the same results were obtained in all studied regimes in a wide range of the spanwise wavenumbers. These data were used then for subsequent processing and obtaining the amplitudes and phases of the distributed vortex-receptivity coefficients.

\section{Procedure of obtaining receptivity coefficients and main results}

The distributed receptivity coefficients are main quantitative characteristics of the distributed receptivity mechanism under study. They were determined in the same manner as in previous work [2] - as the corresponding coefficients of a differential equation that describes the evolution of the distributedly excited TS-modes. An analytical solution of this equation can be written in a general case as:

$$
\begin{gathered}
\bar{B}^{d}\left(x^{*}\right)=e^{\bar{A}\left(x^{*}\right)}\left\{\int_{0}^{x^{*}} \bar{B}_{v}(s) \bar{G}_{v}^{d}(s) e^{-\bar{A}(s)} d s+\int_{0}^{x^{*}} \bar{B}_{v}(s) \bar{G}_{v s}^{d}(s)(\bar{H}(s)+\overline{\bar{H}}(s)) e^{-\bar{A}(s)} d s+\bar{B}_{0}^{d}\right\}, \\
\bar{A}\left(x^{*}\right)=\int_{0}^{x^{*}} \bar{\alpha}(s) d s
\end{gathered}
$$

Here $\bar{B}^{d}$ is the complex-valued amplitude of the boundary layer disturbance; $\bar{\alpha}(x)$ is the $T S$-mode complex-valued wavenumber (obtained from processing of data of the additional, stability experiments); $B_{v}$ is the free-stream vortex complex-valued amplitude (measured at the boundary layer edge); $\bar{H}$ is the surface roughness complex-valued amplitude; $\overline{\bar{H}}$ is its complex conjugate; $\bar{G}_{v}^{d}(x)$ and $\bar{G}_{v s}^{d}(x)$ are the complex-valued distributed vortex- (on smooth surface) and vortex-roughness receptivity functions, respectively; $B_{0}^{d}$ is the complex-valued 'initial' amplitude of the excited boundary layer disturbances and $x^{*}$ is the 
streamwise coordinate with its origin located (for convenience) at the first experiment point of the streamwise region of measurements.

It was assumed in the studied cases that functions $\bar{G}_{v}^{d}(x)$ and $\bar{G}_{v s}^{d}(x)$ are independent of the streamwise coordinate $x$ and correspond to the receptivity coefficients. They were found by means of approximation of the experimental amplitude and phase distributions of the excited boundary layer disturbances $\bar{B}^{d}$ by solution (1) by means of adjustment of unknown solution parameters $\left(\bar{G}_{v}^{d}, \bar{G}_{v s}^{d}\right.$, and $\left.\bar{B}_{0}^{d}\right)$. This fitting was carried out using optimization toolbox of MATLAB (gradient, simplex and genetic algorithms) for two different criteria of deviation of experimental and analytical complex amplitudes. As a result, the receptivity coefficients were obtained in a broad range of parameters.

Fig. $2 a b$ illustrates the amplitude parts of coefficients $\bar{G}_{v}^{d}$ and $\bar{G}_{v s}^{d}$ versus the TS-wave propagation angle $\theta$. Amplitudes $G_{v s}^{d}$ grow with $\theta$ in a monotonous way, while amplitudes $G_{v}^{d}$ depend on $\theta$ in a more complicated way (similar to the experiments [2]). Amplitudes of the two types of receptivity coefficients depend on the task parameters in a quite conservative manner. Amplitudes $G_{v s}^{d}$ obtained in different regimes are very close to each other and sometimes even coincide. The $G_{v}^{d}$ amplitudes also have in different regimes a quite conservative shape of distributions and similar characteristic values. Corresponding estimations showed that if amplitudes of surface roughness $h_{\mathrm{s}} / \delta_{1}$ is less then $\sim 4 \%$, the values of the coefficients of vortex receptivity on smooth surface are higher (in average) then those of the coefficients of the vortex-roughness receptivity. The mechanism associated with the vortex scattering on natural boundary layer nonuniformity dominates in these cases. Meanwhile, the vortex-roughness receptivity mechanism begins to become predominant when the amplitudes of surface waviness become higher than about $4 \%$.
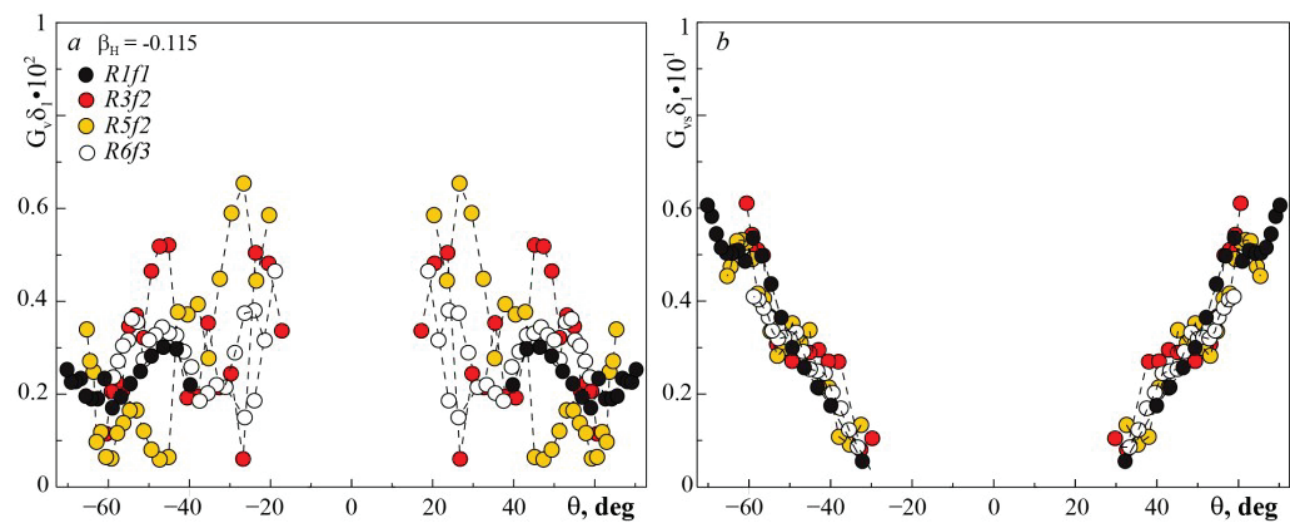

Fig 2. Amplitudes of coefficients of two mechanisms of distributed vortex receptivity: $G_{v}^{d}(a)$ and $G_{v s}^{d}(b)$ obtained in all studied regimes.

Typical results of direct comparison of quantitative receptivity characteristics obtained in the present experiments and in the Blasius boundary layer [2] are shown in Fig. 3 for regimes $R 1 f 1(a, b)$ and $R 3 f 2(c, d)$. It is seen that the amplitude of $G_{v s}^{d}$ depend quite significantly on the streamwise pressure gradient which leads to a noticeable decrease of the receptivity coefficient amplitudes (Figs. $3 b, d$ ). On the contrary, the amplitudes of the receptivity coefficients on smooth surface $\bar{G}_{v}^{d}$ seem to be independent basically of the pressure gradient (Figs. 3a,c). 

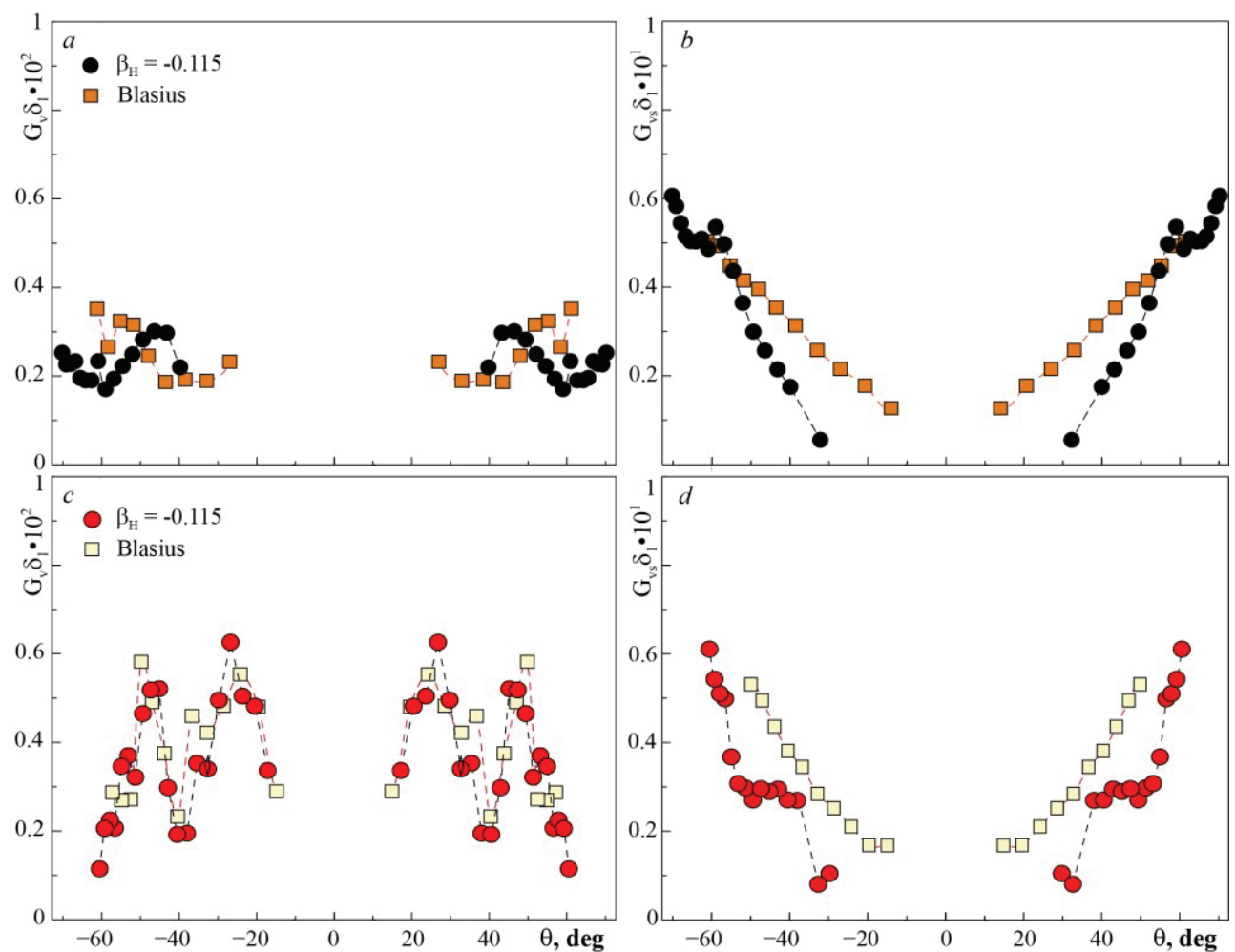

Fig. 3. Comparison of amplitudes of two types of receptivity coefficients in the Blasius flow and in the boundary layer with adverse pressure gradient $\left(\beta_{\mathrm{H}}=-0.115\right)$ for two regimes: $R 1 f 1(a, b)$ and $R 3 f 2$ $(c, d)$

This work is supported by the Russian Foundation for Basic Research, grant No 15-08-07818.

\section{References}

1. V.I. Borodulin, A.V. Ivanov, Y.S. Kachanov, and V.Y. Komarova, Thermophysics and Aeromechanics 13 (2), 199-224 (2006).

2. V.I. Borodulin, A.V. Ivanov, Y.S. Kachanov, and A.A. Fedenkova, Proc. XIII Int. Conf. Methods of Aerophysical Research: Pt III, Publ. House "Parallel", 45-50 (2007) 